\title{
Blue Superluminescent Diode on $c$-Plane GaN Beyond Gigahertz Modulation Bandwidth for Visible Light Communication
}

\author{
Abdullah A. Alatawi1,2, Jorge A. Holguin-Lerma', Chun Hong Kang1, Chao Shen', Abdulrahman M. \\ Albadri' ${ }^{2}$, Ahmed Y. Alyamani², Tien Khee Ng' ${ }^{1}$ and Boon S. Ooi ${ }^{1}{ }^{*}$ \\ ${ }^{1}$ Photonics Laboratory, King Abdullah University of Science \& Technology (KAUST), Thuwal 23955-6900, Saudi Arabia \\ ${ }^{2}$ National Center for Nanotechnology, King Abdulaziz City for Science and Technology (KACST), Riyadh 11442-6086, Saudi Arabia \\ *boon.ooi@kaust.edu.sa
}

\section{Introduction}

Recently, GaN light-emitting diode (LED) and laser-diode (LD) have recently been developed for solid-state lighting (SSL) and visible light communication (VLC) [1, 2]. Superluminescent diode (SLD), which operates in the amplified spontaneous emission (ASE) mode offers further niche in SSL and VLC applications. The ASE (superluminescent) occurs when the optical feedback is suppressed, and thus inhibiting the onset of lasing. SLD offers speckles-free emission that is associated with LD, as well as exhibits shorter carrier lifetime since it operates in the ASE regime. The ASE results in a high modulation bandwidth and high data rate [3], as compared to LED, which operates in the spontaneous emission regime. In our previous work, we have demonstrated $560 \mathrm{MHz}$ [4] and $800 \mathrm{MHz}$ [3] of modulation bandwidth for semipolar-based SLDs. However, the expensive production cost of those substrates prevents them from wide-availability. Thus, here, we demonstrate a $c$-plane GaN-based SLD emitting at $442 \mathrm{~nm}$ with beyond $\mathrm{GHz}$ of bandwidth for simultaneous dual SSL-VLC technology.

\section{Experiments and Results}

At one of the laser facet, a 12-degree tilted waveguide structure was utilized to supress the lasing action. Figure 1(a) shows the electroluminescence (EL) of the SLD at different continuous $(\mathrm{CW})$ injection currents $\left(\mathrm{I}_{0}\right)$. EL confirms the superluminescent characteristic of our SLD as evident in the decreasing FWHM (from $26 \mathrm{~nm}$ to $7 \mathrm{~nm}$ ) as $\mathrm{I}_{0}$ increased. Inset of Fig.1a shows the light-output - current - voltage (L-I-V) characteristics of the superluminescent behaviour characterized by the superlinear dependency of output power on injection currents, with a peak power of $25 \mathrm{~mW}$ at $1 \mathrm{~A}$. Figure 1(b) shows the frequency response of the SLD at different $\mathrm{I}_{0}$ resulting in the achievement of $1 \mathrm{GHz}$ of modulation bandwidth at $-3 \mathrm{~dB}$ (limited by the maximum bandwidth of the Thorlabs APD210 avalanche photodetector). The modulation bandwidth increases with increasing $\mathrm{I}_{0}$, which results in a increasing ASE around $442 \mathrm{~nm}$ with a reduced linewidth of $\sim 7 \mathrm{~nm}$, leading to significantly shorter carrier lifetime [5].
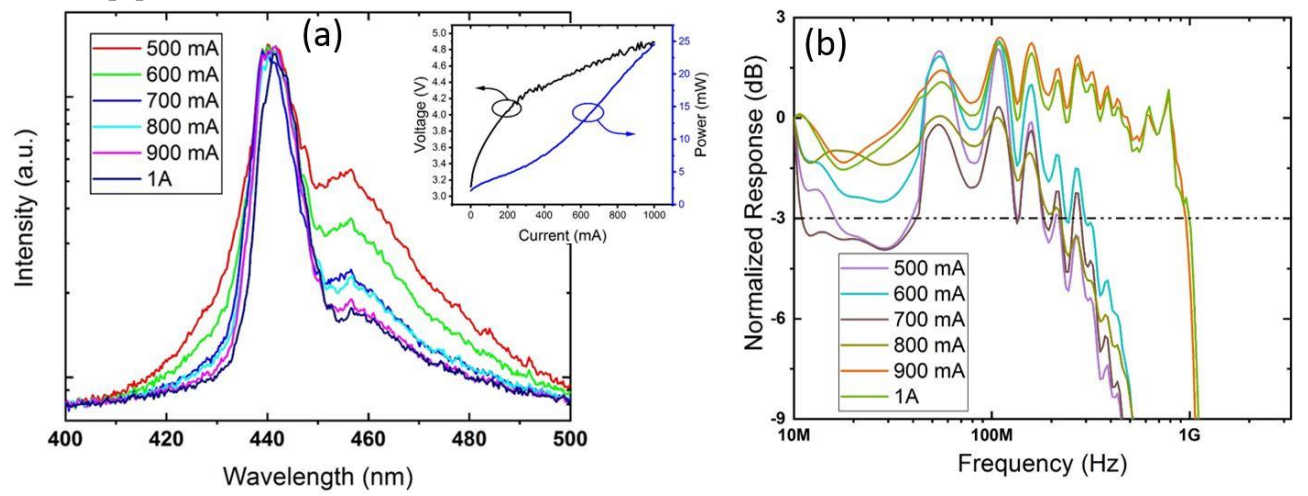

Figure 1: (a) Electroluminescence of the SLD at different $\mathrm{I}_{0}$ with peak emission at $\sim 42 \mathrm{~nm}$. Inset: L-I-V characteristics of SLD with an optical power of $25 \mathrm{~mW}$ at $1 \mathrm{~A}$. (b) The frequency response curves from $500 \mathrm{~mA}$ to $1 \mathrm{~A}$, with the highest bandwidth of $1 \mathrm{GHz}$ at $1 \mathrm{~A}$.

\section{Conclusion}

In conclusion, we have demonstrated a high modulation bandwidth of $1 \mathrm{GHz}$ at $1 \mathrm{~A}$ of injection current for a 442-nm SLD at $25 \mathrm{~mW}$. The $12^{\circ}$ - tilted facet is utilized to supress the onset of lasing. The ASE regime of SLD results in a shorter carrier lifetime, and has led to a high modulation bandwidth, which expands the plethora of devices for applications in SSL-VLC.

\author{
References \\ [1] G. R. Goldberg et al., IEEE Journal of Selected Topics in Quantum Electronics, vol. 23, no. 6, pp. 1-11, 2017. \\ [2] A. A. Alatawi et al., Optics Express, vol. 26, no. 20, p. 26355, 2018. \\ [3] C. Shen et al., Optics Express, vol. 24, no. 18, pp. 20281-20286, Sep 52016. \\ [4] C. Shen et al., Optics Letters, vol. 41, no. 11, pp. 2608-2611, 2016/06/01 2016. \\ [5] R. Hui and M. O'Sullivan, Fiber optic measurement techniques. Academic Press, 2009.
}

